\title{
PRODUÇÃO DE HIPOTERMIA LOCALIZADA NO SISTEMA NERVOSO CENTRAL POR MEIO DE PERFUSÃO DOS VENTRICULOS CEREBRAIS DO CÃO
}

\author{
antonio Carlos Zanini* \\ Regina Oehling * \\ JoÃo TeIXEIRA-PINTO ** \\ LAONE RAMOS*** \\ ARMando Octavio Ramos ***
}

Devido ao interêsse em estudar os efeitos do frio sôbre a excitabilidade do sistema nervoso central, procurou-se estabelecer um método para a produção de hipotermia localizada no encéfalo e a verificação das alterações fisiológicas que disto possam advir. Ademais, sabe-se que a diminuição da temperatura produzida por circulação extracorpórea acarreta sensível redução do volume cerebral, fenômeno êsse a que se atribui importância na terapêutica da hipertensão intracraniana.

A redução da temperatura, restrita ao sistema nervoso central, foi descrita por Golovin ${ }^{1}$, que realizou a perfusão dos $3 .^{\circ}$ e $4 .^{\circ}$ ventrículos cerebrais do cão, com solução de Ringer a $7^{\circ} \mathrm{C}$. Klykov ${ }^{2}$ realizou a perfusão da cisterna magna de cães, obtendo o resfriamento seletivo dos centros bulbares.

\section{MATERIAL E MÉTODO}

Dez cães traqueotomizados ou intubados, anestesiados com pentobarbital sódico (3 $\mathrm{mg} / \mathrm{kg}$, via intravenosa) foram preparados para o registro da respiração e da pressão arterial femoral. A respiração artificial foi praticada, sempre que necessário, por meio de aparelho AGA. Após fixação da cabeça do animal, realizou-se a trepanação bilateral, paramediana, a fim de permitir a colocação, de um lado, de uma cânula de polietileno no ventrículo lateral e, de outro, do eletrodo com cêrca de $3 \mathrm{~cm}$ de comprimento, para o registro da temperatura encefálica. A punção da cisterna magna foi realizada com agulha de punção suboccipital. A temperatura retal foi registrada por meio apropriado.

A perfusão foi efetuada com líqüido de Tyrode, com velocidade variável de 20 a 220 gotas $/$ min. Iniciou-se a perfusão com Tyrode à temperatura de $39^{\circ} \mathrm{C}$, sob

Trabalho do Departamento de Farmacologia (Prof. Charles E. Corbett) e da Clínica Neurológica (Prof. Adherbal Tolosa) da Faculdade de Medicina da Universidade de São Paulo, apresentado no Departamento de Neurologia da Associação Paulista de Medicina em 5 dezembro 1961: * Monitores do Departamento de Farmacologia; ** Assistente extranumerário da Clínica Neurológica; *** Assistentes do Departamento de Farmacologia.

Nota dos autores: Agradecemos aos Srs. Carlos Eduardo P. Corbett e Pedro Carlos Bissetti pela assitência técnica. 
pressão de $150 \mathrm{~mm}$ de água. Cada animal foi, a seguir, submetido a uma ou mais perfusōes a $0^{\circ} \mathrm{C}$, com a duração até de 40 minutos.

\section{RESULTADOS}

A) Modificações da temperatura retal: A temperatura decresceu progressivamente no decorrer de tôdas as experiências até 35 ou $36^{\circ} \mathrm{C}$. Semelhante queda de temperatura foi verificada também em animais simplesmente anestesiados, não submetidos à perfusão dos ventrículos cerebrais.

B) Modificações da temperatura no sistema nervoso central: A temperatura cerebral não sofreu alterações durante a perfusão com Tyrode a $39^{\circ} \mathrm{C}$. Quando se utilizou líqüido perfusor a $0^{\circ} \mathrm{C}$, houve diminuição progressiva da temperatura cerebral até valôres mínimos de $21^{\circ} \mathrm{C}$.

Em alguns animais, na vigência de perfusão fria e depois de estabelecida a hipotermia, verificou-se elevação da temperatura encefálica com tendência a valôres normais.

A reinstalação da perfusão a $39^{\circ} \mathrm{C}$, nos animais que apresentavam hipotermia do sistema nervoso central, resultou em rápida volta da temperatura aos valôres iniciais.

A tabela 1 mostra as variações de temperatura sofridas pelos animais submetidos à perfusão fria.

\begin{tabular}{c|c|c|c|c}
\hline \multirow{2}{*}{ Animal } & $\begin{array}{c}\text { Veloc. de perf. } \\
\text { (gôtas/min.) }\end{array}$ & Temp. cerebral & $\begin{array}{c}\text { Tempo de perfusão } \\
\text { fria }\end{array}$ \\
\hline 1 & 160 & 35,0 & 31,0 & 20 \\
2 & 32 & 35,2 & 26,4 & 40 \\
3 & 80 & 39,0 & 25,0 & 30 \\
4 & 56 & 36,8 & 21,0 & 20 \\
5 & 72 & 38,2 & 28,0 & 15 \\
6 & 146 & 36,6 & 27,0 & 10 \\
7 & 140 & 36,5 & 23,0 & 20 \\
8 & 220 & 38,4 & 27,0 & 15 \\
9 & 85 & 36,6 & 31,0 & 10 \\
10 & 148 & 37,5 & 28,0 & 10 \\
\hline
\end{tabular}

Tabela 1 - Abaixamento da temperatura do sistema nervoso central em cāes submetidos à perfusão dos ventriculos cerebrais com o liqüido de Tyrode a $0^{\circ} \mathrm{C}$ (temperaturas $\mathrm{em}{ }^{\circ} \mathrm{C}$ ). 
C - Modificações da respiração: A perfusão dos ventriculos cerebrais com Tyrode a $0^{\circ} \mathrm{C}$ causou acentuada depressão ou parada da respiração espontânea, de forma a exigir a prática da respiração artificial. O reaquecimento do sistema nervoso central, por meio de perfusão a $39^{\circ} \mathrm{C}$, geralmente propiciou pronto reaparecimento dos movimentos respiratórios espontâneos (fig. 1). Um único animou apresentou recuperação da respiração espontânea durante a perfusão fria.

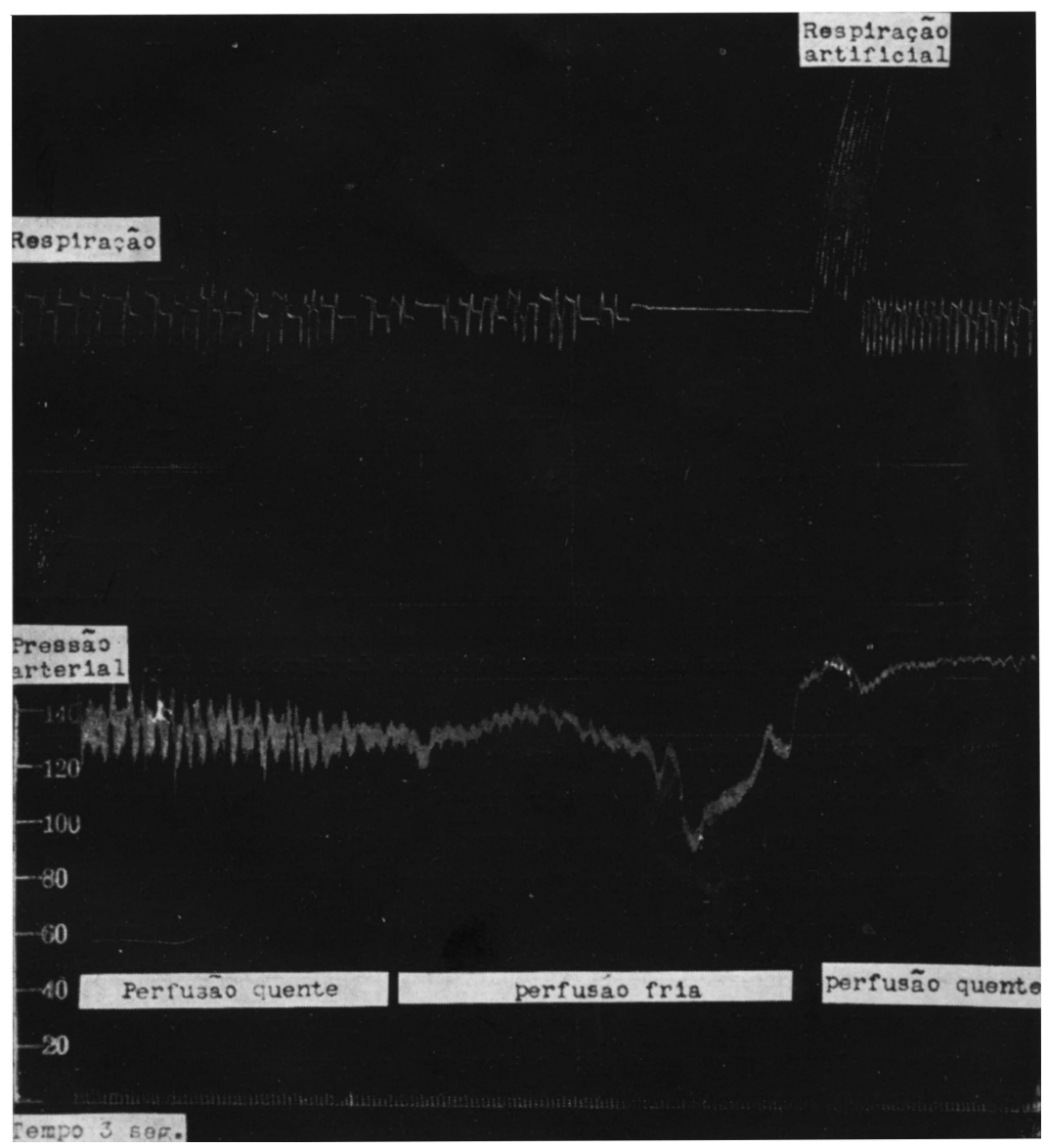

Fig. 1 - Cão de $13 \mathrm{~kg}$, anestesiado pelo pentobarbital sódico $130 \mathrm{mg} / \mathrm{kg}$, via intravenosa). De cima para baixo: registro dos movimentos respiratórios; registro da pressāo arterial femoral; linha $0 \mathrm{mmHg}$ e de marcaçăo de tempo (3 seg); as faixas indicam os periodos de perfusĩo fria (Tyrode $\left.a 0^{\circ} \mathrm{C}\right) \mathrm{e}$ de perfusão quente (Tyrode a $39^{\circ} \mathrm{C}$ ). A perfusão foi realizada com a velocidade de 146 gôtas/min. A temperatura cerebral inicial foi de $36,6^{\circ} \mathrm{C}$, e a mínima, de $27^{\circ} \mathrm{C}$. Quando se deu a parada respiratória, $a$ temperatura cerebral era de $29^{\circ} \mathrm{C}$, e durante a recuperação era de $35,4^{\circ} \mathrm{C}$. 
A tabela 2 mostra as modificações respiratórias observadas nos experimentos realizados.

\begin{tabular}{|c|c|c|c|}
\hline \multirow[b]{2}{*}{ Animal } & \multicolumn{2}{|c|}{ Temp. cerebral $\left({ }^{\circ} \mathrm{C}\right)$} & \multirow[b]{2}{*}{ Observacões } \\
\hline & Parada resp. & Recup. resp. esp. & \\
\hline 1 & 32,0 & - & $\begin{array}{l}\text { Não foi tentada a recuperação da } \\
\text { respiração. }\end{array}$ \\
\hline 2 & 27,0 & 29,2 & - \\
\hline 3 & 32,0 & - & Morte: hemorragia no SNC. \\
\hline 4 & 29,0 & 32,6 & 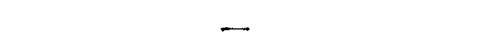 \\
\hline 5 & 29,3 & - & Morte durante perfusão. \\
\hline 6 & 29,0 & 35,4 & - \\
\hline 7 & 28,0 & 34,8 & $\begin{array}{l}\text { Recuperação da respiração duran- } \\
\text { te perfusão fria. }\end{array}$ \\
\hline 8 & 28,0 & 36,8 & - \\
\hline 9 & 33,2 & 34,0 & 一 \\
\hline 10 & 31,2 & - & Morte durante perfusão. \\
\hline
\end{tabular}

D) Modificações cardiovasculares: A perfusão fria dos ventrículos cerebrais influenciou irregularmente a pressão arterial dos animais a ela submetidos. Em alguns, não ocorreu modificação apreciável da tensão sangüínea, enquanto em outros verificou-se hipo ou hipertensão.

Em 3 animais, acompanhando a hipotensão, manifestou-se bradicardia; que desapareceu após a injeção venosa de $1 \mathrm{mg} / \mathrm{kg}$ de sulfato de atropina.

Em outros 2 assinalou-se parada cardiaca (com duração de cêrca de 5 a 6 minutos). Nestes animais, o coração recuperou seus batimentos normais, após a administração intracardiaca de $50 \gamma$ de adrenalina.

\section{DISCUSSAO}

As experiências realizadas mostram a possibilidade de se obter hipotermia localizada no sistema nervoso central. Os animais suportaram variações amplas de temperatura cerebral, enquanto a temperatura retal não foi acentuadamente afetada. 
A função fisiológica mais atingida pela instalação da hipotermia no sistema nervoso central foi a respiração, que foi abolida em tôdas as experiências, exigindo a aplicação de respiração artificial.

De fato, o centro respiratório parece ser bastante sensível à queda de temperatura, pois sua atividade já se mostra deprimida desde os primeiros instantes de perfusão fria e a parada completa da respiração se verificou quando a temperatura cerebral se encontrava o redor de $30^{\circ} \mathrm{C}$. A êsse propósito, é conveniente assinalar que a respiração artificial só foi praticada após depressão muito acentuada ou parada completa da respiração, permanecendo os animais de experiência, desde o início da perfusão fria até a instalação da respiração artificial, em condição de anoxia, que, a nosso ver, pode ser de importância na gênese de certas ocorrências verificadas no decorrer dos experimentos, tais como a hipertensão arterial e a parada cardíaca.

Fato digno de nota é a tendência de elevação da temperatura cerebral, mesmo na vigência de perfusão fria. Tal observação sugere que o organismo põe em jôgo mecanismos compensadores. É provável que, em tais condições, ocorra vasodilatação em áreas cerebrais, a fim de que se processem mais ràpidamente as trocas calóricas entre o sangue e o líqüido de perfusão.

Os resultados que obtivemos mostram uma diferente sensibilidade dos centros nervosos à exposição ao frio. Enquanto o centro respiratório é logo deprimido, os centros vegetativos parassimpáticos parecem ser primeiramente estimulados, pois as hipotensões e bradicardias que foram constatadas foram abolidas pela atropina, indicando dessa forma que êsses fenômenos provàvelmente se devem a estimulação dos centros vagais.

\section{RESUMO}

A perfusão com Tyrode a $39^{\circ} \mathrm{C}$ não ocasionou modificações apreciáveis das temperaturas cerebral e retal. Da mesma forma, não se observaram alterações pronunciadas da respiração e da pressão arterial.

As seguintes alterações foram verificadas com a perfusão a $0^{\circ} \mathrm{C}$ : a) $\mathrm{A}$ temperatura cerebral baixou gradativamente até o nivel mínimo de $21^{\circ} \mathrm{C}$. b) A temperatura retal não foi influenciada pela perfusão fria. As eventuais diminuições observadas, podem ser atribuídas à anestesia, pois ocorreram em cães utilizados para contrôle e, por outro lado, o aquecimento do animal preveniu a referiđa queda de temperatura. c) Depressão e/ou parada respiratória foram fenômenos observados de maneira constante em tôdas as perfusões. A depressão respiratória ocorreu mesmo com temperatura cerebral ao redor de $30^{\circ} \mathrm{C}$. Reiniciadas as perfusões a $39^{\circ} \mathrm{C}$, houve pronta recuperação respiratória. d) Bradicardia, suscetível de ser inibida pela atropina, foi verificada em 3 animais, acompanhando-se de hipotensão arterial. Entretanto, as respostas tensionais foram variáveis: hipotensão ou hipertensão. e) Elevação da temperatura cerebral (com tendência 
a valôres normais), durante a perfusão fria, foi observada em alguns animais. Conquanto não se possa, ainda, explicar convenientèmente o fato, talvez decorra de vasodilatação secundária. f) Em 2 animais ocorreu parada cardiaca, passivel de tratamento pela adrenalina.

\section{SUMMARY}

Localized hypothermia of the brain produced by perfusion through the cerebral ventricles of the dog.

Dogs were anesthetized with sodium pentobarbitone $(30 \mathrm{mg} / \mathrm{kg}$, intravenously). After tracheotomy, the record of the arterial blood pressure was performed, in the femoral artery. The lateral branch of the tracheal cannula was attached to a Marey apparatus. A cannula was planted in the lateral ventricle and other in the cisterna magna. A electrode was also planted in the brain to record the encephalic temperature. Also, the rectal temperature was recorded. The perfusion was performed with Tyrode solution at $0^{\circ} \mathrm{C}$ or $39^{\circ} \mathrm{C}$. The perfusion at $39^{\circ} \mathrm{C}$ did not change the brain or rectal temperature.

The following modifications were verified with perfusion at $\mathrm{O}^{\circ} \mathrm{C}$ : a) The cerebral temperature decreased until $21^{\circ} \mathrm{C}$. No changes were observed in the rectal temperature. b) Respiratory arrest, reversible when the brain was warmed with perfusion at $39^{\circ} \mathrm{C}$. c) Vagal stimulation, with fall of blood pressure and bradycardia, which was abolished by atropine.

\section{REFERENCIAS}

1. GOLOVIN, A. P. - Changes in respiration and blood pressure produced by perfusion of a cold fluid through the cerebral ventricles. In The Problem of Acute Hypothermia. Pergamon, Londres, 1960, pp. 53-61. 2. KLYKOV, N. V. - Changes in respiration and arterial blood pressure produced by the direct effect of low temperature in the bulbar centres. In The Problem of Acute Hypothermia. Pergamon, Londres, 1960, pp. 62-68.

Departamento de Farmacologia - Faculdade de Medicina da Universidade de São Paulo - Av. Dr. Arnaldo - São Paulo, Brasil. 